Revista de Comunicación y Salud, 2020, Vol. 10, n 1, pp. 43-66

Editado por Cátedra de Comunicación y Salud

ISSN: 2173-1675

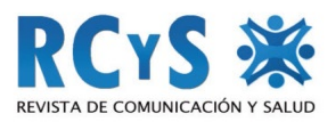

Enviado 24/04/2020

Aprobado 02/06/2020

\title{
BASES PARA EL TRATAMIENTO INFORMATIVO DE LAS DROGAS EN LOS MEDIOS DE COMUNICACIÓN
}

\author{
Carlos A. Ballesteros Herencia ${ }^{1}$ \\ Universidad de Valladolid. España. \\ cballesteros@hmca.uva.es
}

Basis for the informative treatment of drugs in mass media

\section{Resumen}

La responsabilidad social de los medios de comunicación se extiende a todas las materias de que informa, siendo el consumo de drogas un extendido fenómeno social de habitual presencia en la prensa. Por lo que esta investigación se propuso, en primer lugar, describir la representación mediática de las drogas y, en segundo, exponer cómo deberían tratarse informativamente las drogas de un modo responsable desde el doble punto de vista deontológico y preventivo. Para tal fin se analizó la literatura científica y divulgativa sobre este tema hallándose que el tratamiento informativo de las drogas ha sido mayoritariamente criticado por cuantos lo han estudiando en las últimas cinco décadas. Los medios de comunicación ofrecerían una información en ocasiones sensacionalista y habitualmente no contrastada, ya que tiende a utilizarse una única fuente, mayoritariamente institucional, que resulta de fácil acceso para el periodista. Además se ha acusado a los medios de propagar estereotipos, difundir una imagen imprecisa, cuando no errónea, de este fenómeno y hasta de desempeñar un papel contrapreventivo. Pese a ello, se ha destacado la importancia para la prevención de contar con la colaboración de los periodistas y los medios de comunicación, en tanto éstos son la principal fuente de información sobre drogas para la población general. La aparición de Internet ha sumado un nuevo campo de investigación sobre comunicación y drogas de múltiples formatos, canales y contenidos, donde coexisten población general, jóvenes, instituciones y profesionales de los medios.

Palabras clave: medios de comunicación, periodismo, ética, deontología profesional, drogas, prevención.

\section{Abstract}

The social responsibility of the media extends to all matters it reports on, such us drug use, a widespread social phenomenon with a regular presence in the press. Therefore,

\footnotetext{
${ }^{1}$ Autor para correspondencia: Carlos A. Ballesteros Herencia cballesteros@hmca.uva.es
} 
this research tried, firstly, to describe the media representation of drugs and, secondly, to expose how drugs should be informatively treated in a responsible manner from a double deontological and preventive point of view. For this purpose, the scientific and informative literature on this topic was analyzed, finding that the informative treatment of drugs has been mostly criticized by those who have studied it in the last five decades. The media offers information that is sometimes sensationalist and usually not verified, since a single source, mostly institutional, tends to be used, which is easily accessible to the journalist. In addition, mass media have been accused of spreading stereotypes, an imprecise, if not mistaken, image of this phenomenon and even playing a counterpreventive role. Despite this, the important collaboration of journalists and the media has been highlighted, as these are the main source of information on drugs for the general population. The emergence of the Internet has added a new field of research on communication and drugs of multiple formats, channels and content, where the general population, youth, institutions and media professionals coexist.

Keywords: mass media, journalism, professional ethics, deontology, drugs, prevention.

\section{Cómo citar el artículo}

Ballesteros Herencia, C. A. (2020). Bases para el tratamiento informativo de las drogas en los medios de comunicación. Revista de Comunicación y Salud, 10(1),43-66. doi: http://doi.org/10.35669/rcys.2020.10(1).43-66

\section{INTRODUCCIÓN}

\subsection{La relevancia social de las drogas}

El término "drogas" recibe múltiples usos, como reconoce la Organización Mundial de la Salud (World Health Organizatión, 2003). Mientras en el ámbito de la medicina se refiere "a toda sustancia con potencial para prevenir o curar una enfermedad, o de aumentar el bienestar físico o mental, en farmacología se aplica a cualquier agente químico que altera los procesos fisiológicos" (OMS, 1994). Popularmente, el uso del término "drogas" tiende a restringirse a aquellas sustancias de comercio ilegal y uso recreativo con efectos psicoactivos. Sin embargo la Organización Mundial de la Salud ha puntualizado que tabaco, alcohol o cafeína también son drogas, y que la mayor carga sobre la salud global es producida precisamente por las sustancias legales, ya que el $43 \%$ de la población mundial adulta (2.348 millones de personas) consume alcohol (World Health Organizatión, 2018) y un 25\% consumen tabaco (1.337 millones) (World Health Organizatión, 2019).

En España la tercera parte de la población adulta admite haber consumido en algún momento de su vida alguna sustancia ilícita, mientras el alcohol lo ha consumido el $91,2 \%$, el tabaco el $69,7 \%$ e hipnosedantes el 20,8\%. Las drogas ilegales más consumidas son el cannabis $(35,2 \%)$ y la cocaína $(10,2 \%)$ (PND, 2019).

Revista de Comunicación y Salud, 2020, Vol. 10, n 1, pp. 43-66 
La preocupación de la población española hacia las drogas ha sufrido un gran descenso en las últimas cuatro décadas (ver datos en gráfico 1), pasando de ser considerado el segundo mayor problema en el año 1988, cuando era citado por casi la mitad de los españoles, a prácticamente desaparecer en la década de los años 2010, con menos del $1 \%$ que las cita (CIS, 2020).

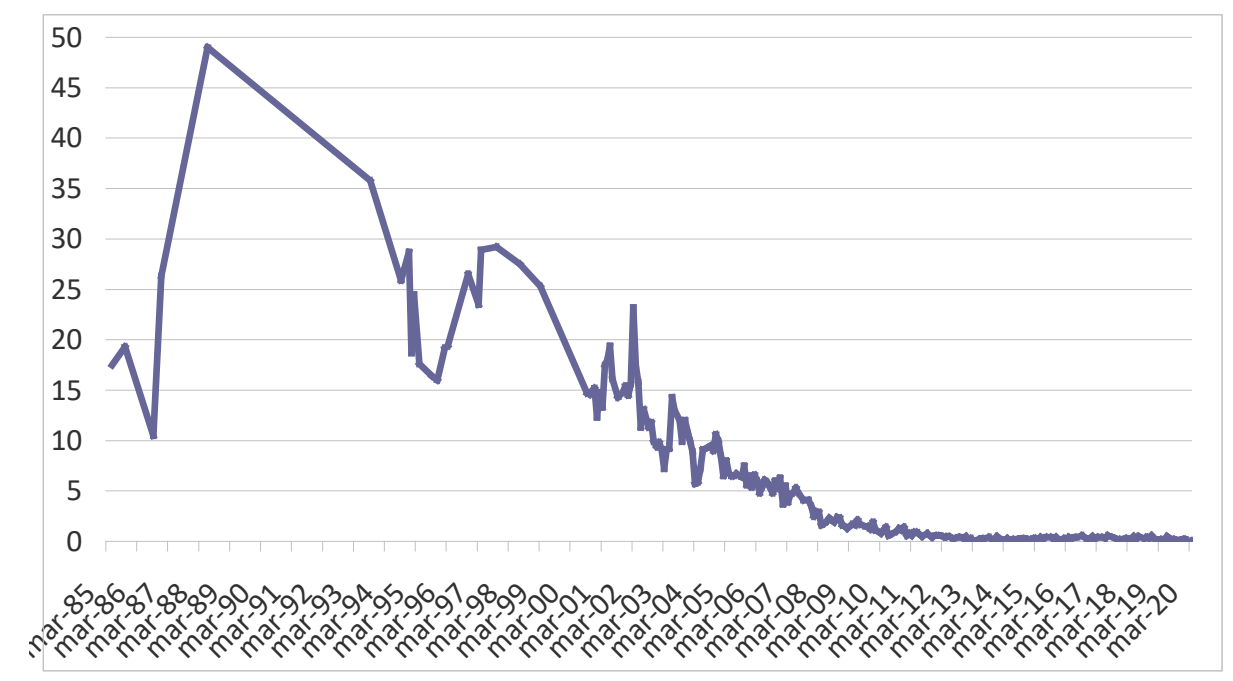

Gráfico 1. Porcentaje de españoles que citan las drogas como uno de los principales problemas.

Fuente: CIS (2020), elaboración propia.

Esta tendencia a la baja en la preocupación de los españoles hacia las drogas no se corresponde con la evolución de su consumo. Así en los últimos 20 años, el consumo de alcohol y tabaco se ha mantenido estable (Ver datos en Gráfico 2), mientras que ha crecido el de las drogas ilegales más consumidas, por ejemplo el del cannabis del 22 al $30 \%$, y el de la cocaína de 3,4 al 10\%. El consumo de heroína, droga que mayor alarma despertó en los años 80, se ha mantenido estable en torno al 0,6\% (PND, 2019).

Revista de Comunicación y Salud, 2020, Vol. 10, n 1, pp. 43-66 
Bases para el tratamiento informativo de las drogas en los medios de comunicación

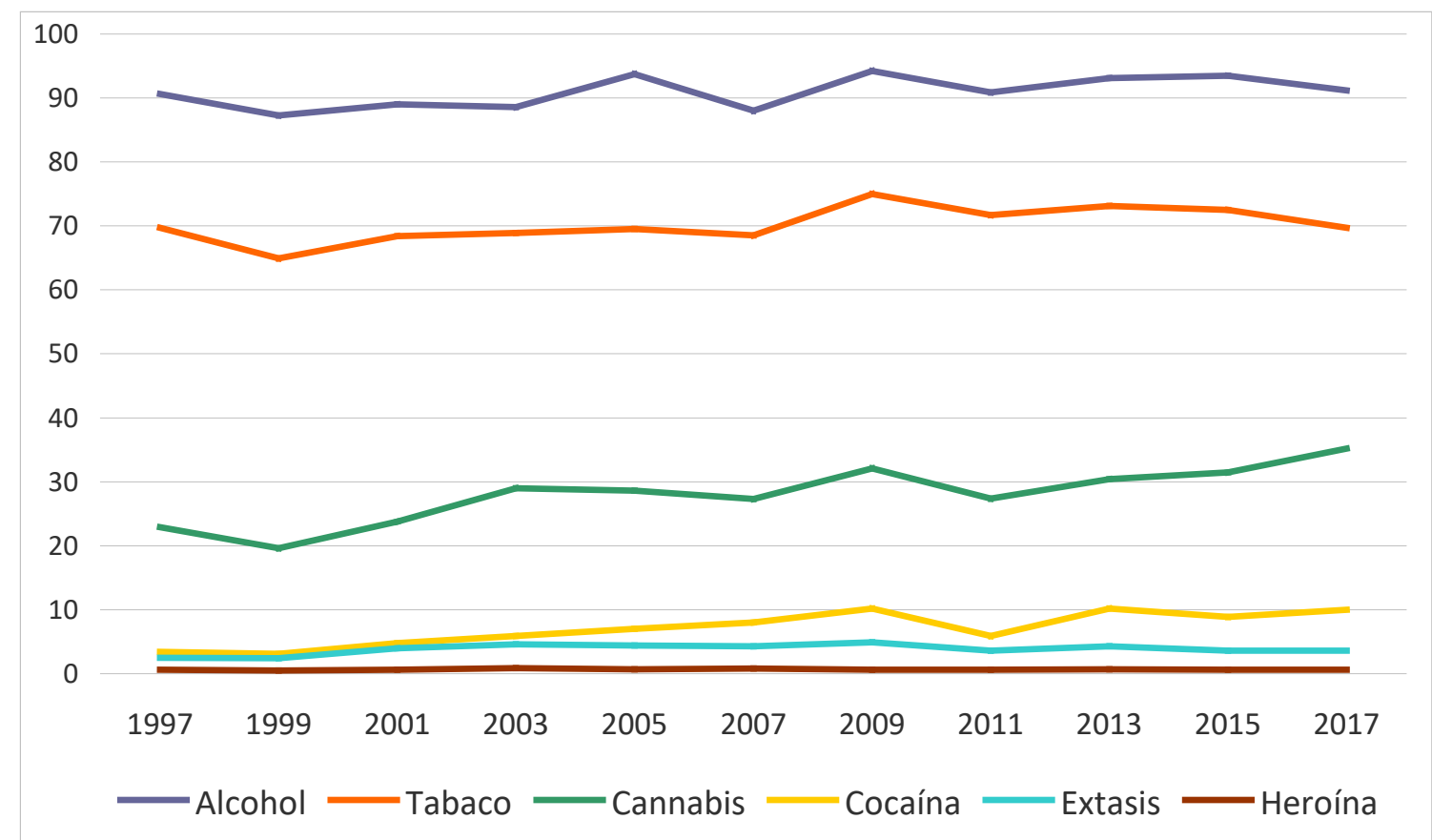

Gráfico 2. Consumo de drogas alguna vez en la vida (1997-2017).

Fuente: PND (2019), elaboración propia.

\section{OBJETIVOS}

Ante tal importancia del fenómeno social de las drogas esta investigación se propuso como objetivo principal determinar cuáles son las características de un tratamiento informativo responsable de las drogas en los medios de comunicación. Para lograr este propósito se planteó en primer lugar explorar y analizar la literatura científica y divulgativa sobre la representación mediática de la drogas. Para ello se trató de describir las características formales y de contenido de las informaciones sobre drogas en la prensa española, así como profundizar en el impacto de las nuevas tecnologías en la relación con las drogas. Finalmente nos propusimos sintetizar las recomendaciones propuestas en publicaciones académicas y preventivas para transmitir una imagen acertada de las drogas.

\section{LA REPRESENTACIÓN DE LAS DROGAS EN LOS MEDIOS DE COMUNICACIÓN}

La responsabilidad de los medios de comunicación ante las drogas es una cuestión habitual entre la literatura científica y divulgativa sobre este tema (p.e. Del Olmo, 1997; Becoña, 2002; Junta de Castilla y León, 2008). Ya en 1995, la Organización de las Naciones Unidas consideraba "de capital importancia" la colaboración de los medios de comunicación social para la reducción de la demanda, instando a gobiernos y a medios de comunicación a adoptar campañas y políticas "que impidan que se fomente el

Revista de Comunicación y Salud, 2020, Vol. 10, nº 1, pp. 43-66 
Bases para el tratamiento informativo de las drogas en los medios de comunicación

consumo no terapéutico de drogas" (Junta Internacional de Fiscalización de Estupefacientes, 1995, p.6)

Esta recomendación ha sido traspuesta en los ámbitos nacionales, de modo que en España la comunicación ha sido considerada como una de las seis áreas transversales de actuación que conformaban la Estrategia Nacional sobre Adicciones 2009-2016 (PND, 2017). Esta estrategia reconocía el "protagonismo de los medios de comunicación" en la prevención, junto a los sistemas sanitario y social (PND, 2009, p. 66), de modo que el Plan Nacional sobre Drogas (PND, 2017) describía cómo se estaba realizando por parte de administraciones y organizaciones no gubernamentales una importante labor informativa y sensibilizadora en distintos contextos, citando entre ellos a los medios de comunicación, redes sociales o páginas web.

No en vano, la fuente principal de información sobre drogas para la población general son los medios de comunicación (56,9\%), muy por delante de conocidos $(26,1 \%)$ o profesionales sanitarios (8,7\%) (PND, 2017). Por ello Becoña (2002) en sus "Bases científicas de la prevención de drogodependencias", reclama "desarrollar la conciencia de comunidad y de los medios de comunicación de masas en relación con las drogas".

Autores como González (1992) o Vega (1996) coinciden en que las drogas son un tema frecuente en los medios de comunicación, de modo que el primero habla de "bombardeo informativo recurrente de sucesos" (p. 33), mientras el segundo lo califica de "inflación informativa sobre las drogas" (p.117).

Desde que en 1973 la Unesco afirmase que "los efectos producidos por los medios de información han sido más negativos que positivos" (p. 2), no han dejado de acumularse las críticas contra la representación mediática de las drogas, calificada como "sensacionalista" en numerosas ocasiones (Del Olmo, 1997; PND, 2000; Rekalde y Romaní, 2002; Perales y Del Pueyo, 2019; Zaragoza y Elgueta, 2019). Es ésta una acusación que se ha mantenido en el casi medio siglo que ha transcurrido desde el clásico informe de la Unesco de 1973, donde se estimaba que se tiende "a rodear el tema de una aureola indeseable y de un sensacionalismo indebido cuyos efectos, en algunos casos, son casi equivalentes a los de un manual de instrucciones para administrar drogas" (p. 2), hasta la actualidad, cuando Perales y Del Pueyo (2019) siguen sosteniendo que el sensacionalismo "distorsiona negativamente la prevención de consumos".

Autores como Berjano et al. (1990), Usó (1995), Del Olmo (1997), Martín (2010) o Perales y Del Pueyo (2019) coinciden en que la información mediática sobre drogas produciría efectos contrapreventivos. De este modo, Berjano et al. (1990) afirman que la información sobre drogas "en algunas ocasiones, constituye un mecanismo incitador" del consumo (p. 189). Adicionalmente, se ha acusado a los medios de comunicación de ofrecer una imagen distorsionada, de contribuir a la desinformación o de realizar un tratamiento incorrecto (p.e. Berjano et al, 1990; Vega, 1996; Del Olmo, 1997; García et al., 2011).

Revista de Comunicación y Salud, 2020, Vol. 10, n 1, pp. 43-66 
Se ha señalado a varios factores como causa de esta situación. Por un lado a los criterios clásicos de noticiabilidad (actualidad, proximidad, consecuencias, rareza, conflicto, suspense, emoción, entre otros según Carl Warren, 1979), que priorizarían los aspectos dramáticos, delictivos y de conflicto (González, 1992, p.91), y por otro a las características propias del medio. Esto coincide con la clasificación en factores periodísticos y extraperiodísticos que guían el proceso de elección de contenidos informativos que realiza Ortells-Badenes (2014). Entre estos últimos criterios González (1992) asegura que la propia "lógica mercantilista que inspira la tarea de las empresas informativas hace que los profesionales tiendan a la espectacularización y dramatización de la noticia" (p. 79).

A la misma naturaleza de los medios de comunicación también se refiere el manual "Medios de comunicación y drogodependencias" editado por el Plan Nacional sobre Drogas al señalar que "las limitaciones de tiempo en los medios audiovisuales, o de espacio en los escritos, predisponen a generalizar" (PND, 2000).

En este sentido, las actuaciones de represión se ajustarían a la inmediatez e impacto "que requieren las crónicas de sucesos. Sin embargo, los actos relacionados con la prevención y la asistencia al problema de las drogas son, por naturaleza, lentos y sin el aparente brillo que tienen las noticias sobre drogas centradas en alijos o muertes" (PND, 2000, p. 20).

Pero también se ha incidido en que el tratamiento inadecuado de las drogas en los medios se ha debido a la falta de especialización y formación de los periodistas (Junta de Castilla y León, 2008; Martín, 2010; Gayo, 2013; PND, 2015). En este sentido el Plan Nacional sobre Drogas (2015) detectaba "falta de especialización y conocimiento del tema" en el ámbito periodístico por lo que recomendaba "acometer la especialización y formación en el conocimiento del problema de las adicciones".

También la Red Iberoamericana de ONG's que trabajan en Drogodependencias (Martín, 2010) señalaba que la formación académica de los periodistas no incluye ningún apartado sobre educación en salud "ni mucho menos sobre drogodependencias, por lo que es necesario hacer un esfuerzo para suplir esa carencia" (p.14). Igualmente desde el ámbito institucional, la Junta de Castilla y León (2008) describía cómo la mayoría de los profesionales de la información "no conocen a fondo el tema de las drogodependencias, sus fuentes son escasas y parciales, trabajan a contrarreloj y no tienen posibilidad de especializarse" lo que desembocaría en que los medios estarían favoreciendo una imagen criminalizada de las drogas y actitudes consumistas hacia las sustancias (p.21).

Finalmente, Gayo (2013) entiende que la ausencia de especialización contribuye a la desinformación, ya que "poquísimos licenciados en Ciencias de la Información, por no decir ninguno, están interesados hoy en día en formarse en la información sobre drogas. Como mucho, abordarán el tema desde la óptica de los sucesos, algo muy 
Bases para el tratamiento informativo de las drogas en los medios de comunicación

extendido en los medios" (p. 246). Por su parte González (1992) también sostenía la necesidad de esta especialización en drogas, independientemente que se adquiera mediante un título de máster o experto "o simplemente mediante la práctica profesional".

\subsection{Aspectos formales y campos temáticos}

En el aspecto formal, dos estudios sobre la imagen de las drogas en la prensa escrita durante los años 80 y 90, el de García et al. (1987) y el de Martínez (2000) coincidían en sus resultados. Las informaciones sobre drogas en la prensa se presentaban principalmente mediante el género de la noticia, tendían a ser breves, no ocupaban lugares destacados, carecían de apoyo gráfico y no contrastaban la información.

Una década más tarde los resultados del estudio de Núñez, Paricio y Rodríguez (2012) siguen confirmando que el modelo informativo vigente sobre las drogas no difiere del de hace décadas en España "ya que prima la información poco profunda, las temáticas de tráfico de drogas, la ubicación de los textos en las secciones relacionadas con los sucesos y el recurso a fuentes policiales para elaborar la información" (p.30).

En el caso concreto del cannabis Ballesteros (2014) también encuentra que éste se representa en la prensa escrita con escasa importancia, no se contrastan las informaciones y las noticias suelen ser enmarcadas mediante el frame de conflicto. Estos resultados son corroborados por Santos y Camacho (2017) quienes detectan que el tratamiento que recibe esta sustancia se caracteriza por un estilo eminentemente informativo, "lo que indica que no existe una preocupación por redactar textos elaborados, que profundicen y ofrezcan explicaciones y opiniones" (p. 165).

Además existe consenso en publicaciones que abarcan casi cinco décadas en que el campo temático preferente para informar sobre drogas es el delictivo (Unesco, 1973; García et al., 1987; González-Zorrilla, 1987; González, 1992; Rekalde y Romaní, 2002; Pantoja y Abeijón, 2004; Martín, 2010; Serena, 2010; Paricio, Núñez y Rodríguez., 2011; Núñez et al., 2012; Ballesteros, 2012; Santos y Camacho, 2017; Zaragoza y Elgueta, 2019). Se ha considerado que esta orientación informativa al narcotráfico se ha olvidado de "la realidad de las personas afectadas" (Rekalde y Romaní, 2002, p.25), dando de lado a los programas comunitarios y a los consumidores (VVAA, 1991, p. 165), y llegándose a la conclusión ya expuesta por la Unesco en 1973 de que el hincapié de los medios en el aspecto punitivo resultaba contraproducente en cuanto a la prevención (p.2).

De esta forma más de la mitad de las veces que los medios de comunicación se ocupan de las drogas lo hacen para informar sobre aspectos delictivos (p.e. García et al., 1987; Núñez et al., 2012; Ballesteros, Muñiz y Dader, 2015; Santos y Camacho, 2017) quedando en un segundo plano los ámbitos políticos y legislativo (por debajo del $30 \%$ ), y el científico-medio y el social (siempre por debajo del 10\%). Según Paricio el al. (2011) esta orientación temática hacia el delito y la represión se produce "a pesar de los

Revista de Comunicación y Salud, 2020, Vol. 10, n 1, pp. 43-66 
Bases para el tratamiento informativo de las drogas en los medios de comunicación

esfuerzos institucionales y políticos por situar las drogas como un problema social y sanitario" (p. 71). Según Martín (2010) la dimensión sociosanitaria del consumo de drogas sigue sin tener una presencia significativa en medios, con la excepción del tabaco y el uso terapéutico del cannabis.

En consonancia con este encuadre delictivo, se produce una orientación negativa de estas informaciones (Froján et al., 1993; PND, 2000; Rekalde y Romaní, 2002; Zaragoza y Elgueta, 2019; Perales y Del Pueyo, 2019).

Pese a esta escasa variación temática a lo largo del tiempo la Red Iberoamericana de ONG que trabajan en Drogodependencias afirma que hay cada vez menos referencias que asocien drogadicción y marginalidad (2010), mientras que Perales y Del Pueyo (2019), aseguran que se observa una mayor presencia mediática del punto de vista sociosanitario.

\subsection{Fuentes informativas y protagonistas de la información}

Se ha encontrado un extendido acuerdo (Oliva, 1986; García et al, 1987; González, 1992; Froján et al, 1993; PND, 2000; Martínez, 2000; Rekalde y Romaní, 2002; Markez, 2003; Núñez et al., 2012; Perales y Del Pueyo, 2019; Zaragoza y Elgueta, 2019) sobre el protagonismo que los actores y las fuentes institucionales, especialmente los cuerpos de seguridad del Estado, interpretan en las informaciones sobre drogas. En cambio, las fuentes y actores sanitarios y sociales son secundarios o no aparecen (Zaragoza y Elgueta, 2019).

En este sentido, el Plan Nacional sobre Drogas advierte que emplear una sola fuente para informar sobre drogas limita la comprensión este fenómeno (PND, 2000), y que el periodista, emulando la multidisciplinariedad de los equipos que se ocupan de las drogodependencias, debería acudir a una mayor variedad de expertos, como farmacólogos, psiquiatras, psicólogos, asistentes sociales, sociólogos, o pedagogos.

Esta fuente única suele ser de tipo institucional según una mayoría de investigaciones (Oliva, 1986; García et al, 1987; Rekalde y Romaní, 2002; Márkez, 2003; Paricio et al, 2011; Ballesteros, 2012, 2014; Perales y Del Pueyo, 2019), y más concretamente de tipo policial o judicial, a la que se ha llegado a calificar de "fuente única periodística" en el tema de las drogas. Los periodistas en lugar de contrastar las informaciones o buscar noticias originales se limitarían "a recoger las notas de prensa, declaraciones de personajes y autoridades, versiones oficiales, entrevistas, informes, dossieres de documentación y ese tipo de productos de comunicación que preparan las oficinas de prensa institucionales" (Rekalde, 2002, p.32). Con una relevancia intermedia, las fuentes sanitarias y científicas oscilan entre el 15 y el $30 \%$ según los diferentes estudios.

Finalmente, en el otro lado de la balanza los protagonistas afectados por las drogas, los consumidores y los colectivos sociales apenas logran el estatus de fuentes

Revista de Comunicación y Salud, 2020, Vol. 10, n 1, pp. 43-66 
Bases para el tratamiento informativo de las drogas en los medios de comunicación

informativas (Rekalde, 2002; Ballesteros, Dader y Muñiz, 2015; Perales y Del Pueyo, 2019). Curiosamente, las pocas veces en que se ha empleado a los consumidores como fuentes se ha hecho de tal modo que una publicación del PND (2000) advertía de que recoger el testimonio de un drogodependiente puede resultar "peligroso si no se matiza, bien porque transmita que si se puede salir por qué no probar, o por reflejar la imagen de un sujeto pasivo, sin responsabilidad sobre su adicción" (p.23), por lo que se aconseja seleccionar cuidadosamente los testimonios de consumidores, ya que "las opiniones de estas personas en ningún caso constituyen evidencias científicas. No por el hecho de ser o haber sido consumidor de drogas o drogodependiente se tiene razón en el enfoque o en la solución del problema" (PND, 2008).

Junto a esta fuente policial, parecen haberse ido abriendo camino las instituciones que trabajan en drogas, como recogen tanto Paricio el al. (2011) como Perales y Del Pueyo (2019). Estos últimos destacan la relevancia alcanzada por el Plan Nacional sobre Drogas, así como de las organizaciones especializadas como la Asociación Proyecto Hombre, la FAD, Energy Control, UNAD o PDS.

En paralelo con estas fuentes, los actores protagonistas del relato mediático sobre drogas son también las instituciones, entre las resaltan los cuerpos y fuerzas de seguridad del Estado y la Justicia, "que hablan de lo que ellos mismos hacen, y de a quién se lo hacen" (Perales y Del Pueyo, 2019).

Finalmente conviene destacar la conveniencia de citar la fuente siempre que sea posible, ya que las fuentes permiten avalar la fiabilidad de los mensajes, evitando expresiones como "críticas vecinales al nuevo centro asistencial" o "Los especialistas están preocupados por el incremento del consumo de marihuana", ya que estas frases pueden hacer que se interprete como evidencias científicas lo que no son sino apreciaciones subjetivas (PND, 2008). Igualmente los periodistas deben verificar la fiabilidad de las fuentes de información, recabando la opinión de expertos de diferente ámbitos extremando el rigor en el lenguaje y el tratamiento de los datos (JCYL, 2008). Conviene destacar que estudios como el de García et al. (2011) señalan que las fuentes más creíbles para los jóvenes universitarios en materia de drogas son las revistas de divulgación científica y los libros divulgación, mientras que entre los medios de comunicación destaca la prensa escrita frente al último lugar de Internet.

\subsection{Generación de estereotipos}

La escasa reelaboración periodística de la información proveniente de fuentes institucionales ha originado una serie de estereotipos frecuentes sobre las drogas (González, 1992; González-Zorrilla, 1987; Berjano et al., 1990; Usó, 1995; PND, 2000; Rekalde y Romaní, 2002; JCYL, 2008; PND, 2008; Serena, 2010)

En primer lugar enmarcar las drogas como un delito ha producido una equiparación entre consumidor de drogas y delincuente. En parte ha podido deberse a la ausencia de los consumidores no problemáticos como fuente de información, sustituidos por las

Revista de Comunicación y Salud, 2020, Vol. 10, nº 1, pp. 43-66 
Bases para el tratamiento informativo de las drogas en los medios de comunicación

fuentes policiales, obviándose que una mayoría de los consumidores están integrados en la sociedad, la familia y el trabajo (PND, 2000). Este estereotipo del consumidordelincuente ha ido evolucionando hacia otro de consumidor-enfermo que necesita atención sanitaria (Rekalde y Romaní, 2002).

Esta identificación del consumidor de drogas como "drogadicto" y "delincuente" se produce únicamente para referirse al consumidor de drogas ilegales. En cambio la imagen mediática de los consumidores de drogas legales es la de un individuo normalizado, integrado, que sólo causa conflicto cuando conduce un automóvil bajo los efectos del alcohol (Martínez, 2000).

Un segundo estereotipo consistiría en utilizar el singular "droga" o bien hablar de "drogas" en general (PND, 2015) en lugar de referirse a diversas sustancias con efectos diferentes. Frente a la advertencia de que cada sustancia "requiere un tratamiento específico" (PND, 2008), "en el tratamiento informativo de las drogas, todo es una misma cosa" (González-Zorrilla, 1987). El origen de este reduccionismo se remonta a los años 80 y la alarma social creada en torno a la heroína.

Según describe el Plan Nacional sobre Drogas (2000) al hablar de la droga, en singular, "estamos lanzando un mensaje codificado que el lector u oyente interpreta como "la heroína" y todo lo que ésta conlleva: marginalidad, delincuencia, inseguridad ciudadana, etc." (p. 22). Sin embargo esta interpretación parece haberse transmitido a nuevos contextos, de modo que Santos y Camacho (2017) detectan en representación mediática actual del cannabis "una concepción monolítica de todos los tipos de droga, según la cual la droga es una sola y sus efectos son los mismos".

Este estereotipo ha producido el empleo habitual de la expresión "drogas, tabaco y alcohol", que el manual "Medios de comunicación y drogodependencias" editado por Plan Nacional de Drogas (2000) estima "especialmente inadecuada" (p.22), ya que contribuiría a difundir la idea de que el alcohol y el tabaco no son drogas. En su lugar, habría que referirse al "tabaco, alcohol y otras drogas".

Otros estereotipos que han sido detectados identifican y asocian de un modo reduccionista a jóvenes con drogas, heroína con marginalidad, cannabis con progresismo, éxtasis con diversión y discotecas, o cocaína con éxito social (PND, 2008, 2015) lo que provocaría una visión desenfocada y simple de cada una de estas complejas realidades.

\subsection{Algunos equívocos habituales}

Junto a lo ya descrito se puede comprobar la coincidencia de la literatura académica y divulgativa en advertir de una serie de desaciertos frecuentes por parte de los medios de comunicación en la información sobre drogas (Santacreu et al., 1992; Froján et al., 1993; Usó, 1995; Vega, 1996; PND, 2000, 2008, 2009, 2015; Markez, 2003; JCYL, 2008) y que podemos sintetizar en cuatro grandes epígrafes.

Revista de Comunicación y Salud, 2020, Vol. 10, nº 1, pp. 43-66 


\subsubsection{Jóvenes y drogas}

Se ha trasmitido una imagen que identifica alcohol con fiesta y ésta con drogas, mediante escenas de fiestas juveniles en las que se sirven y se consumen bebidas alcohólicas. Se transmite la idea del consumo de drogas como algo consustancial con la juventud, como si ésta fuera un colectivo homogéneo.

En ocasiones se emplea un lenguaje institucional que no cala entre los jóvenes al no utilizar sus mismos códigos de comunicación. Emplear un tono paternalista, infantilizador o meramente prohibicionista que se limita a decir a los jóvenes lo que no deben hacer termina por causar rechazo a los mensajes preventivos. Por añadidura utilizar la generación de miedo hacia las drogas como estrategia, puede transmitir un mensaje que contradice la experiencia personal de los sujetos, que terminarán por desconfiar de estas fuentes.

\subsubsection{Naturaleza de las drogas}

En primer lugar, a la hora de informar sobre el consumo de drogas, resulta más preciso hablar de consumos en plural, ya que se ha impuesto lo que se denomina policonsumo, esto es, tomar a la vez varias sustancias psicoactivas. Además el consumo de drogas puede ser diferente dependiendo del momento y de la persona:

- El consumo esporádico es frecuente entre la población general y puede estar desprovisto de consecuencias significativas para la salud. Sin embargo restarle importancia a cualquier forma de consumo de drogas contribuye a normalizarlo y banalizarlo. En el caso de los jóvenes este consumo esporádico tiene un carácter experimental, de descubrimiento.

- El consumo problemático se caracteriza por una intensidad, un entorno o una edad de los consumidores, que puede generar un problema de salud significativo.

- En el caso de la drogodependencia, la adicción hace que los consumidores enfoquen parte de su actividad diaria en el consumo de una o varias drogas, y en asegurar unos consumos que se convierten en reiterativos y necesarios.

En segundo lugar, se ha transmitido el mensaje de que las drogas producen en todos los casos dependencia y adicción casi instantánea y por igual en todos los consumidores.

Por otro lado, se ha generalizado atribuir a las sobredosis la causa de las muertes de drogodependientes, olvidando que las causas más comunes suelen ser la adulteración y las enfermedades "oportunistas".

Revista de Comunicación y Salud, 2020, Vol. 10, nº 1, pp. 43-66 
En el extremo contrario, en ocasiones se magnifica el potencial terapéutico de ciertas drogas como si de panaceas se tratasen, olvidando limitaciones, contraindicaciones y efectos secundarios que puedan presentar, y que determinados usos terapéuticos no equivalen a que el consumo recreativo resulte inocuo.

\subsubsection{Narcotráfico}

La imagen que se ha transmitido en muchas ocasiones del narcotráfico y su represión también ha sido cuestionable. En primer lugar al informar continua y repetidamente de "golpe al narcotráfico" ha terminado por extrañar que éste se siga produciendo, lo que solo conduce a dos explicaciones lógicas: o bien se exagera al utilizar estos términos o bien se genera la idea de que se esté produciendo un crecimiento incontrolado de los grupos delictivos.

Insistir en el valor que puede alcanzar la mercancía decomisada en el mercado negro podría inducir a cometer nuevos delitos entre ciertos sectores desfavorecidos y faltos de recursos.

Al mismo tiempo se tiende a cuantificar la sustancia decomisada de un modo que resulte llamativo para el público, de modo que se utilizan unidades de medida heterogéneas: toneladas en el caso del hachís, kilos de cocaína o miles de dosis si son papelinas de heroína. Relacionado con la extensión en los últimos años del cultivo de la marihuana se ha popularizado entre los medios la expresión "plantaciones", en ocasiones para referirse a un pequeño número de plantas cultivadas en un jardín o una maceta.

\subsubsection{Lenguaje y formato periodístico}

La utilización del lenguaje conlleva la transmisión de asociaciones automáticas que el profesional de la información ha de controlar. Por ejemplo, se ha utilizado reiteradamente la expresión "drogas de diseño", que podría transmitir una connotación atractiva, siendo más preciso referirse a drogas sintéticas o de laboratorio.

Por otra parte la utilización de términos despectivos como "drogatas", "yonkis", "enganchados", etc. o de expresiones exitosas en un determinado momento como "el azote de las drogas" o "caer en el pozo de la droga", suelen transmitir mensajes moralistas y catastrofistas que en poco ayudan a formar una imagen realista.

En cuanto al contenido visual de las noticias, es frecuente utilizar imágenes de archivo con una relativa relación con el mensaje que se transmite. Se debe intentar que la imagen corrobore el mensaje verbal, evitando por el contrario contribuir a fijar estereotipos como los descritos. Frente al empleo de imágenes criminalizadoras, degradantes o morbosas de las personas consumidoras es posible seleccionar imágenes de profesionales e iniciativas de prevención.

Revista de Comunicación y Salud, 2020, Vol. 10, nº 1, pp. 43-66 
Bases para el tratamiento informativo de las drogas en los medios de comunicación

\subsection{Internet y redes sociales}

La preocupación por la información sobre drogas que pudiera difundirse a través de Internet es expresada ya en 1998 por la Asamblea General de Naciones Unidas, que recomendó a los medios de comunicación tradicionales y a las industrias de telecomunicaciones y de producción de programas informáticos promover el autocontrol para eliminar información ilegal en materia de drogas (ONU, 1998).

Unos años después, en 2002, encontramos esta preocupación expresada en España por Becoña, quien reclama atención "por la relación que individuos vulnerables puede tener con las drogas, el incremento de información a través de la red, la obtención de drogas en ella, etc." (p.110). Así, el Plan Nacional sobre Drogas (2015) considera a las redes sociales y las nuevas tecnologías de la información como "los mejores vehículos para aportar información sobre drogas a la población, no solo por su alcance sino por sus costes más reducidos" (p. 18) por lo que recomienda a todo el sector que trabaja en la prevención desarrollar estrategias de comunicación en Internet para contrarrestar los mensajes contrapreventivos que aparecen en la red. Las nuevas tecnologías proporcionan nuevos retos a la vez que "avances en las estrategias de la comunicación y la salud" que han de contar con "la participación activa del propio receptor" (Cuesta y Menéndez, 2011). Como ejemplo del intento por actualizarse y seguir el ritmo del cambio tecnológico desde los ámbitos institucionales se puede citar el objetivo del PND en su Plan de Acción sobre Adicciones 2018-2020 por lanzar una APP dirigida principalmente a informar sobre drogas y adicciones al público general (Ministerio de Sanidad, 2018).

La revisión sistemática realizada por Mukherjee et al. (2019) afirmaba que todavía existe muy poca información sobre los usos, beneficios y limitaciones de los medios sociales para la comunicación para la salud. Es éste un tema crucial pues como asegura Taffe (2015) la búsqueda en Internet de información relacionada con la salud se ha convertido el comportamiento predeterminado de las personas, incluyendo el uso recreativo y abuso de sustancias psicotrópicas. Al Khaja, Al Khaja y Sequeira (2018) detectaron en su análisis de mensajes distribuidos a través de Whatsapp que la mayoría de los mensajes relacionados con las drogas en las redes sociales eran afirmaciones potencialmente engañosas o falsas que carecían de evidencia creíble para respaldarlas.

En el lado preventivo, Gountas et al. (2014) encontraron que los vídeos subidos a las redes sociales podían contribuir positivamente en el cambio de actitudes y en reducir el consumo excesivo de alcohol.

Mackey, Liang y Strathdee (2013) también se refieren a "estas tecnologías no reguladas" como un riesgo potencial en lo que respecta a la experimentación juvenil con medicinas no prescritas por personal médico. Según estos autores Internet y los medios sociales como Facebook o Twitter son un punto crítico de acceso de los jóvenes a estas drogas. Esto es así, porque las redes sociales son utilizadas por muchos adolescentes como medio predominante o exclusivo para informarse, lo que puede generar falsas expectativas sobre los efectos de la alcohol y otras drogas (Stankova, 2020).

Revista de Comunicación y Salud, 2020, Vol. 10, nº 1, pp. 43-66 


\subsection{La prensa antiprohibicionista}

Más allá de los medios generalistas, existe un tipo de prensa especializada, que se podría definir como "antiprohibicionista" o más específicamente "cannábica", pues se enfoca en todo lo relacionado con el consumo y el cultivo del cannabis.

En España han existido diversas revistas "cannábicas" de distribución nacional, desde la decana El Cogollo (1997), a otras como Cáñamo, Cannabis Magazine, Yerba (edición española de la estadounidense High Times) o Soft Secrets (de la editorial holandesa Discover Publisher). Cáñamo es la revista de referencia de este mercado, cuyo control de difusión OJD en 2002 (único disponible) arrojaba una tirada de 48.750 ejemplares. Bobes y Calafat (2000) la describían como "moderna, bien editada y cuenta con diversas colaboraciones de profesionales entusiastas y vinculados a diferentes facetas de la amplia cultura juvenil, como la música, el cine, los espectáculos, etc." (p. 246).

El "Informe sobre el cánnabis" (GEC, 2004) consideraba que estas publicaciones y la proliferación de sitos web constituían una de las causas del aumento del consumo de cannabis. De un modo más constructivo algunos autores resaltan la capacidad de llegar a los consumidores de estas publicaciones. De este modo, Romaní (citado en Markez, 2003, p.107) señala que "hay gente a la que le interesa lo que allí se dice, porque saben que es un tipo de información que parte de unas premisas mucho más cercanas a la realidad (...). Y estoy seguro de que las contraindicaciones en relación a ciertos usos del cannabis que en algunas ocasiones allí se han publicado, llegan mucho mejor a los interesados (que las de los discursos oficiales)".

También Bobes y Calafat (2000) destacan que estas revistas y asociaciones podrían realizar "una gran contribución social si ayudaran a crear los criterios de prevención que nuestra sociedad necesita, con el fin de impedir el aumento del consumo de cannabis entre individuos que aún están en proceso de formación". En este sentido el editorial del primer número de La María de Soft Secrets (2002) se comprometía a "informar con veracidad, asumiendo la responsabilidad de difundir las estrategias de reducción del daño y una máxima precaución por la protección integral del menor" (p. 271).

\section{La responsabilidad de los medios ante las drogas}

Los medios de comunicación juegan un importante papel ante todo tipo de problemas sociales, entre los que podemos situar el fenómeno del consumo de drogas. Según la "Teoría de la responsabilidad social de la prensa", elaborada por la Comisión sobre la Libertad de Prensa o Comisión Hutchins (1947), correspondería a los medios "proporcionar una explicación verdadera, comprensiva e inteligente de los acontecimientos diarios dentro de un contexto en el que adquieran significado" (Commission, 1947, p. 20). 
En concreto, los códigos deontológicos, en tanto deudores de esta teoría, aportan una guía mayoritariamente aceptada sobre cómo deben realizar su tarea informativa los periodistas. Pese a que la Unesco (1973) alentaba ya tempranamente a las organizaciones profesionales de información "a incluir normas sobre la utilización de los datos sobre el uso indebido de estupefacientes en sus respectivos códigos deontológicos" (p. 5) según Gayo (2013) son pocos son los medios de comunicación que incluyen en sus códigos o libros de estilo alguna referencia acerca de cómo tratar la información sobre las drogas. En este sentido, el Manual de Estilo de RTVE (2011) realiza dos breves apuntes. Por un lado la indicación genérica de que "en la información sobre consumo de sustancias nocivas, permitidas o no por la ley, no debe animarse a su consumo". Respecto a programas infantiles se asevera que "estos contenidos solo tienen razón de ser cuando se trate de prevenirles sobre los riesgos de estas sustancias".

La Federación de Asociaciones de Periodistas de España (FAPE, 2017), define el ejercicio profesional del periodismo como un importante compromiso social, ya que permite el desarrollo de los derechos fundamentales sobre la libre información y expresión de las ideas.

Según la FAPE el respeto a la verdad se constituye como un compromiso ético del periodista. La libertad de investigar y difundir con honestidad la información son dos deberes esenciales. Junto a ello la información sobre los menores de edad debe ser realizada con especial atención.

Finalmente el Código de la FAPE establece que el periodista debe asumir el principio de que toda persona es inocente mientras no se demuestre lo contrario, y evitar al máximo las posibles consecuencias dañosas derivadas del cumplimiento de sus deberes informativos. Tales criterios son especialmente exigibles cuando la información verse sobre temas sometidos al conocimiento de los Tribunales de Justicia. Dentro del Estatuto del Periodista de la FAPE podemos destacar los siguientes puntos:

- Derecho y deber a una formación profesional actualizada y completa (8.c).

- Corresponde al periodista vigilar escrupulosamente el cumplimiento por parte de las Administraciones Públicas de su obligación de transparencia informativa (11).

- Deberá fundamentar las informaciones que difunda, lo que incluye el deber de contrastar las fuentes y de dar la oportunidad a la persona afectada de ofrecer su versión de los hechos (13.a).

Coincide el Código Deontológico del Colegio de Periodistas de Cataluña (Col.legi, 1992) al afirmar que sólo deben difundirse informaciones fundamentadas, evitando datos imprecisos que puedan socavar la dignidad de las personas y provocar un daño o descrédito injustificados a instituciones y entidades públicas y privadas. 
Bases para el tratamiento informativo de las drogas en los medios de comunicación

\section{Conclusiones}

El consumo de todo tipo de drogas, ya sean legales como el alcohol, el tabaco o los fármacos, o ilegales como el cannabis, la cocaína y los productos sintéticos, es un fenómeno socialmente generalizado, que ha despertado preocupación en ámbitos oficiales, académicos y sociales. Sin embargo la revisión bibliográfica realizada coincide en que los medios de comunicación no observan los criterios profesionales en sus informaciones sobre drogas (Unesco, 1973; Vega, 1996; Del Olmo, 1997; PND, 2000; Rekalde y Romaní, 2002; Perales y Del Pueyo, 2019; Zaragoza y Elgueta, 2019), sino que han creado una imagen equívoca que podría contribuir a provocar situaciones contrapreventivas y a fomentar el abuso de sustancias (Berjano et al., 1990; Usó, 1995; Del Olmo, 1997; Martín, 2010). Podríamos sintetizar el tratamiento periodístico actual de las drogas en dos grandes características.

Por un lado las informaciones sobre drogas no se contrastan, sino que se suelen publicar, con escasa reelaboración periodística, las notas de prensa institucionales, principalmente policiales (García et al, 1987; Rekalde y Romaní, 2002). Paradójicamente, esta reproducción inalterada del punto de vista de una única fuente otorga una apariencia de objetivismo a lo que en realidad es una información no contrastada. La incorporación en los últimos años de las fuentes sanitarias y preventivas ha añadido al estereotipo clásico del consumidor-delincuente, la imagen del consumidor de drogas como un enfermo, obviando a la amplia mayoría social de personas consumidoras de drogas legales e ilegales que llevan una vida normalizada.

En segundo lugar la naturaleza de las drogas y el fenómeno social que su consumo ha generado se describe de modo confuso. Por ejemplo no se diferencia entre los diferentes tipos de drogas, empleándose de modo genérico tanto el singular "la droga", identificándola con la heroína, y transmitiendo toda la carga simbólica de esta droga a las demás sustancias (PND, 2000) como el plural "las drogas", transmitiéndose una imagen similar de todas ellas. Junto a esto, en ocasiones no se considera como drogas a las sustancias de comercio legal (PND, 2001; Megías et al., 2001).

Para rematar esta imagen, los medios habrían aportado un valor añadido simbólico a las drogas, dotándolas de un significado de pertenencia a grupo (García et al., 1987), al relacionar su consumo con estilos de vida, identidad, etc. (Gobierno Vasco, 1994). Además en ocasiones se ha podido suscitar la curiosidad de los jóvenes y hasta descubrir nuevas vías de administración (VVAA, 1991; Usó, 1995). Resaltar las ganancias del tráfico de drogas ha podido animar a delinquir a ciertos sectores sociales desfavorecidos (PND, 2000; Usó, 1995).

Frente a tan dudoso tratamiento informativo, la responsabilidad de los medios periodísticos ante las drogas ha sido demandada reiteradamente por instituciones e investigadores, y aparece claramente establecida tanto en normas generales, como los códigos deontológicos, como en guías específicas, manuales e investigaciones que se han elaborado sobre este asunto. Es más, muy diversos estudios coinciden en la

Revista de Comunicación y Salud, 2020, Vol. 10, n 1, pp. 43-66 
Bases para el tratamiento informativo de las drogas en los medios de comunicación

capacidad de los medios de comunicación para difundir campañas de prevención y sensibilizar frente al consumo abusivo de drogas (García et al., 1987; Costa y Pérez, 1989; Vega, 1996; Berrio, 2000; PND, 2000; Rekalde y Romaní, 2002; Pantoja y Abeijón, 2004; Paricio, Núñez y Rodríguez, 2012).

Para lograrlo se ha propuesto una mayor colaboración entre periodistas y profesionales de la prevención, por ejemplo en forma de jornadas y elaboración conjunta de campañas preventivas (González, 1992; PND, 2009). También se ha subrayado la necesidad de dotar de una mayor especialización en este tema a los periodistas (González, 1992; PND, 2009; Junta de Castilla y León, 2008; Martín, 2010; Gayo, 2013; PND, 2015).

Independiente y complementariamente de esta mayor formación los periodistas cuentan con un importante instrumento para mejorar la información sobre drogas, como son los códigos deontológicos. En primer lugar aplicándose con total diligencia, y salvando los obstáculos de la precariedad laboral y los recortes de plantillas, al deber de reproducir verazmente los hechos y de ejercer críticamente la profesión. Y sobre todo contrastando las fuentes, una de las críticas más extendidas y a la vez uno de los principios más conocidos de la profesión, presente en todos los códigos deontológicos y libros de estilo.

En este sentido parece haberse moderado la "fuente única policial" dominante en la España de los años 80 y 90, y haberse atendido en parte la demanda por una mayor atención a las dimensiones sanitarias y sociales que lograra describir mejor el contexto de los hechos. Así, se ha detectado cómo se ha evolucionado hacia una progresiva y creciente consideración de las fuentes preventivas institucionales (Paricio el al., 2011; Perales y Del Pueyo, 2019). Lograr tener en cuenta, equilibrada y contrastadamente, también a los colectivos sociales y a los consumidores podría terminar por mostrar todo el panorama de este complejo fenómeno social.

Esta ampliación del contexto también pasa por prestar atención a todas las sustancias, independientemente de su estatus legal, ya que son precisamente las drogas legales las más consumidas y las que mayor perjuicio causan a la sociedad (World Health Organization, 2018, 2019). Equilibrar la atención entre drogas legales e ilegales debe partir de considerar que el consumo de cualquier sustancia con potencial adictivo no es inocuo (PND, 2009)

Un caso muy específico es el de la prensa antiprohibicionista o cannábica, publicaciones que en la terminología del informe MacBride (1988, p. 296) podríamos denominar como "comunicaciones de sustitución y de contrainformación", ya que realizan una reinterpretación de la información oficial proveniente de las instituciones. Como señala este clásico informe "reevalúan y extienden las comunicaciones en función de una nueva concepción de su importancia en la sociedad". Su inicial formato impreso pronto se multiplicó en innumerables sitios de Internet que tratan las drogas desde el punto de vista del consumidor recreativo. Las redes sociales han atraído el interés de los investigadores sobre el papel de la comunicación en el consumo de

Revista de Comunicación y Salud, 2020, Vol. 10, nº 1, pp. 43-66 
Bases para el tratamiento informativo de las drogas en los medios de comunicación

drogas, generándose en poco tiempo una importante investigación con muy diferentes perspectivas (p.e. Al Khaja, Al Khaja y Sequeira, 2018; Mackey, Liang y Strathdee, 2013; Gountas et al., 2014; Taffe, 2015; Mukherjee et al., 2019).

Tal profusión de información sobre las drogas por todo tipo de canales promovida por todo tipo de autores no ha hecho sino acentuar la inflación informativa de la que se hablaba aún antes de la eclosión digital. Ya Abraham Moles hacía notar que "las personas disponen de muchos más instrumentos y contenidos de comunicación de los que jamás llegarán a poder utilizar" (Moles 1986, p. 151). Resulta preocupante que esta superabundancia informativa pueda contribuir a una mayor distorsión de la imagen del fenómeno social de las drogas. Para que los ciudadanos sean social e individualmente responsables necesitan disponer de una información adecuada. Por tanto, no parece que se hayan corregido las clásicas acusaciones hacia la imagen mediática de las drogas, a la vez que el nuevo panorama tecnológico precisa de una atención investigadora renovada y actualizada capaz de la complicada tarea de monitorizar su evolución en tiempo real.

\section{REFERENCIAS}

Al-Khaja, K.A.J., Al-Khaja, A.K., Sequeira, R.P. (2018). Drug information, misinformation, and disinformation on social media: a content analysis study. Journal of Publich Health Policy. 39(3), 343-357. doi: http://doi.org/10.1057/s41271-018-0131$\underline{2}$

Ballesteros, C. A. (2012). Los marcos informativos del cannabis en la prensa española: aplicación de las teorías del" framing" y de la" agenda-setting" (Tesis doctoral). Universidad Complutense de Madrid.

Ballesteros, C. (2014). El enmarcado informativo del cannabis: un estudio cuantitativo exploratorio desde la teoría del framing. Anuario Electrónico de Estudios en Comunicación Social "Disertaciones", 7(1), 67-103.

Ballesteros, C., Dader, J.L. y Muñiz, C. (2015). Los marcos informativos del cannabis en la prensa española. Evidencia empírica desde la teoría del framing. Zer-Revista de estudios de comunicación, 20(38), 67-85.

Becoña, E. (2002) Bases científicas de la prevención de drogodependencias. Madrid: Ministerio del Interior. Delegación del Gobierno para el Plan Nacional sobre Drogas.

Berjano, E., Gracia, E., García, F. y Musitu, G. (1990). Imagen de las drogas en los medios de comunicación social. En G. Musitu (Coord). Procesos psicosociales básicos (pp.189-94.). Barcelona: Promociones y Publicaciones Universitarias.

Revista de Comunicación y Salud, 2020, Vol. 10, nº 1, pp. 43-66 
Bases para el tratamiento informativo de las drogas en los medios de comunicación

Berrio, J. L. (2000). Medios de comunicación y drogas. En X. Arana, A. Vega, A. y I. Markez (Eds.). Drogas: cambios sociales y legales ante el tercer milenio (pp. 219234). Madrid: Dykinson.

Bobes, J. y Calafat A. (eds.) (2000). Monografía Cannabis. Adicciones, 12 (2). Recuperado de 2007 desde http://www.mir.es/pnd/observa/pdf/cannabis.pdf

CIS (2020). Tres problemas principales que existen actualmente en España. Recuperado de http://www.cis.es/cis/export/sites/default/Archivos/Indicadores/documentos html/TresProblemas.html

Col.legi de Periodistes de Catalunya (1992). Código Deontológico. Barcelona.

Commision (1947). A free and responsible press. A General Report on Mass Communication: Newspapers, Radio, Motion Pictures, Magazines, and Books. The Commission on freedom of the press. Chicago: The University of Chicago. Recuperado de http://www.archive.org/download/freeandresponsib029216mbp/freeandresponsib0292 $16 \mathrm{mbp} . \mathrm{pdf}$

Costa, P.O. y Pérez, J.M. (1989). Droga, televisión y sociedad. Madrid. Plan Nacional sobre Drogas.

Cuesta, U. y Menéndez, T. (2010). Drogas, comunicación y nuevas tecnologías de la información. Observatorio de drogodependencias de Castilla-La Mancha, (6), 93-104.

Del Olmo, R. (1997). Los medios de comunicación social y las drogas. Comunicar: Revista científica iberoamericana de comunicación y educación, (9), 119-124. Recuperado de https://dialnet.unirioja.es/descarga/articulo/634168.pdf

Diezhandino, P. (2009). Criterio noticioso. El quehacer periodístico ante el desafío digital. Madrid: Pearson.

FAPE (2017). Código Deontológico. Recuperado de http://fape.es/home/codigodeontologico/

Froján, M.J., Rubio, R. y Peris, B. (1993). La imagen de la droga en la prensa española. Un análisis de la información publicada entre 1988 y 1992. Revista española de drogodependencias, 3, 191-204.

García, J.A., Gásquez, M. y García, A. (2011). Importancia de la credibilidad de las fuentes mediáticas en materia de drogas. Miguel Hernández Communication Journal, 2, 173-196.

Revista de Comunicación y Salud, 2020, Vol. 10, n 1, pp. 43-66 
Bases para el tratamiento informativo de las drogas en los medios de comunicación

García, B., Menor J. y Perales, A. (1987). La imagen de la droga en la prensa española. Madrid: Plan Nacional sobre Drogas.

Gayo, A. (2013). El papel de los medios de comunicación: más fuentes, más rigor, más debate... menos riesgos. En D. Martínez y J. Pallarés (Eds), De riesgos y placeres. Manual para entender las drogas (p. 143-154). Lleida: Editorial Milenio.

GEC (2004). Informe sobre el cánnabis 2004: Análisis de situación y propuestas de actuación. Madrid: Grupo de Estudios sobre el Cánnabis.

Gobierno Vasco (1994). La opinión pública vasca ante la legalización de la venta de drogas. San Sebastián: Administración de la Comunidad Autónoma del País Vasco. Secretaría de la Presidencia del País Vasco.

González, N. (1992). Estudios sobre droga y medios de comunicación en España. Comunicación y sociedad, 5(1-2), 79-101. Recuperado de https://revistas.unav.edu/index.php/communication-and-society/article/view/35540

González-Zorrilla, C. (1987). Drogas y control social. Revista poder y control, 2, 49-65.

Gountas, S., Ciorciari, J., Gountas, J. y Huddle, S. (2014). An investigation of the EEG correlates associated with viewing alcohol and drug related behaviours on social media. En 17th World Congress of Psychophysiology of the International Organization of Psychophysiology, (pp. 251-251). Hiroshima: Elsevier. doi: http://doi.org/10.1016/j.ijpsycho.2014.08.956

JCYL (2008). Código de Buenas Prácticas para el tratamiento informativo en materia de drogodependencias. Valladolid: Junta de Castilla y Léon. Comisionado Regional para la Droga.

Junta Internacional de Fiscalizacion de Estupefacientes (1995). Eficacia de los tratados de fiscalización internacionales de drogas. Nueva York: Naciones Unidas. Recuperado de https://www.incb.org/documents/Publications/AnnualReports/AR1994/E-INCB-19941-Supp-1-s.pdf

MacBride, S. (1988). Un solo mundo, voces múltiples. Comunicación e información en nuestro tiempo. Madrid: Fondo de Cultura Económica.

Mackey, T.K., Liang, B.A., Strathdee, S.A. (2013). Digital Social Media, Youth, and Nonmedical Use of Prescription Drugs: The Need for Reform. Journal of Medical Internet Research, 15(7). doi: http://doi.org/10.2196/jmir.2464

Martín, J. (2010). Buenas prácticas de los medios de comunicación en materia de drogas. Red Iberoamericana de ONG que trabajan en Drogodependencias (RIOD).

Revista de Comunicación y Salud, 2020, Vol. 10, nº 1, pp. 43-66 
Bases para el tratamiento informativo de las drogas en los medios de comunicación

Recuperado de https://riod.org/wp-content/uploads/2017/05/11.-2010Buenaspr\%C3\%A1cticas-de-los-MMCC-en-materia-de-drogas-RIOD-web.pdf

Martínez, J. (2000). El discurso social sobre drogas. (Tesis Doctoral). Universidad de Murcia, España.

Megías, E. (Dir.) (2001). Los valores de la sociedad española y su relación con las drogas. Madrid: Fundación La Caixa.

Moles, A. (1986). Théorie structurale de la communication et société. París: Éditions Masson.

Oliva, M.P. (1986). Los medios de comunicación social ante las drogodependencias. Comunidad y Drogas, 1, 65-73.

Ministerio de Sanidad (2018) Plan de Acción sobre Adicciones 2018-2020. Plan Nacional sobre Drogas. Recuperado de http://www.pnsd.mscbs.gob.es/pnsd/planAccion/docs/PLAD 2018-2020 FINAL.pdf

Mukherjee, S.K., Kumar, J., Jha, A.K., y Rani, J.R. (2019). Role of Social Media Promotion of Prescription Drugs on Patient Belief-system and Behaviour. International Journal of e-collaboration, 15(2), 23-43. doi: http://doi.org/10.4018//JeC.2019040102

OMS (1994). Glosario de términos de alcohol y drogas. Madrid: Ministerio de Sanidad.

ONU (1998) Sesión Especial de la Asamblea. Organización de las Naciones Unidas. Recuperado de http://www.pnsd.mscbs.gob.es/delegacionGobiernoPNSD/relacionesInternacionales/ ONU/declaracion/home.htm

Núñez, F., Paricio, P., y Rodríguez, C. (2012). 'Framing' y morfología en el tratamiento Periodístico de las drogas. Index Comunicación, (2), 13-36. Recuperado de: http://journals.sfu.ca/indexcomunicacion/index.php/indexcomunicacion/article/v $\underline{\text { iew/25/25 }}$

Pantoja, L. y Abeijón, J.A. (coord.) (2004). Los medios de comunicación y el consumo de drogas. Avances en drogodependencia. Bilbao: Universidad de Deusto.

Paricio, P., Núñez, F. y Rodríguez, C. (2011). Fuentes, temas y encuadres en la información sobre drogas en la prensa española. El caso de El País, El Mundo, ABC y La Razón. Revista de Comunicación, 10, 71- 101.

Paricio, P., Núñez, F. y Rodríguez, C. (2012). Comunicación, prevención del consumo de drogas y opinión pública: El tratamiento informativo de las drogas en ABC, El País

Revista de Comunicación y Salud, 2020, Vol. 10, no 1, pp. 43-66 
Bases para el tratamiento informativo de las drogas en los medios de comunicación

y El Mundo (2009-2010). En M.D. Cáceres y A. Lucas (Eds.), Crisis y cambios en la sociedad contemporánea: comunicación y problemas sociales (pp. 109-127). Madrid: Fragua.

Perales, A. y Del Pueyo, B. (2019). El tratamiento de las drogas en los medios de comunicación. Proyecto Hombre: revista de la Asociación Proyecto Hombre, 98, 2429. Recuperado de http://archivo.proyectohombre.es/revista-proyecto/el-tratamientode-las-drogas-en-los-medios-de-comunicacion/

PND (2000). Medios de comunicación y drogodependencias. Actuar es posible, 9. Madrid: Plan Nacional sobre Drogas. Ministerio de Sanidad.

PND (2009) Estrategia Nacional sobre Drogas. 2009-2016. Plan Nacional sobre Drogas. Recuperado de http://www.pnsd.mscbs.gob.es/pnsd/estrategiaNacional/docs/EstrategiaPNSD20092016.pdf

PND (2015). Plan de Comunicación del Plan Nacional sobre Drogas. Prioridades de los mensajes, las poblaciones diana y canales comunicativos a utilizar para prevenir el consumo de sustancias psicoactivas. Plan Nacional sobre Drogas. Recuperado de http://www.pnsd.mscbs.gob.es/pnsd/planAccion/plan/productos/pdf/Accion 3 docum ento consenso.pdf

PND (2017). Estrategia Nacional sobre Drogas 2009-2016. Informe de la evaluación final. Ministerio de Sanidad. Plan Nacional sobre Drogas. Recuperado de http://www.pnsd.mscbs.gob.es/pnsd/estrategiaNacional/docs/2017 Informe Evaluaci on Final Estrategia Nacional sobre Drogas 2009 2016.pdf

PND (2019). Estadísticas 2019. Alcohol, tabaco y drogas ilegales en España. Ministerio de Sanidad. Recuperado de http://www.pnsd.mscbs.gob.es/profesionales/sistemas/nformacion/informesEstadistic as/pdf/20190EDA-ESTADISTICAS.pdf

Rekalde, A. y Romaní, O. (2002). Los medios de comunicación social ante el fenómeno de las drogas: un análisis crítico. San Sebastián. Servicio Central de Publicaciones del Gobierno Vasco.

RTVE (2011). Manual de Estilo de RTVE. Recuperado de http://manualdeestilo.rtve.es/cuestiones-sensibles/5-16-tabaco-alcohol-y-otras$\underline{\text { drogas/ }}$

Santos, M.T. y Camacho, I. (2017). El tratamiento del cannabis en la prensa española. Cuadernos.Info, 40, 153-171. https://doi.org/10.7764/cdi.40.980

Revista de Comunicación y Salud, 2020, Vol. 10, nº 1, pp. 43-66 
Bases para el tratamiento informativo de las drogas en los medios de comunicación

Serena, F. (2010). Cuando los medios de comunicación hablan de drogas. En II Congreso Internacional de Investigación y Práctica Profesional en Psicología (pp. 303-305). Buenos Aires: Universidad de Buenos Aires.

Taffe, (2015) Drug abuse scientists should use social media to engage the public because their primary translational product is information. Drug and Alcohol Dependance, 154(1), 315-319. doi: http://doi.org/10.1016/i.drugalcdep.2015.05.012

Unesco (1973). Reunión de especialistas sobre los métodos para evaluar la acción de los grandes medios de información contra el uso indebido de estupefacientes, informe final. Recuperado de https://unesdoc.unesco.org/ark:/48223/pf0000003404 spa.locale=es

Usó, J.C. (1995) Drogas y Cultura de masas (España 1855-1995). Madrid: Taurus.

Vega, A. (1996). ¿Los medios de comunicación educan sobre las drogas? Comunicar, 6. Recuperado de https://www.revistacomunicar.com/ojs/index.php/comunicar/article/view/C06-1996-23

VVAA (1991). Medios de comunicación en el "problema drogas" y la percepción de soluciones. Madrid: Plan Nacional sobre Drogas.

Warren, C. (1979). Géneros periodísticos informativos. Barcelona, ATE.

World Health Organizatión (2018). Global status report on alcohol and health 2018. Recuperado de https://apps.WorldHealthOrganizatión.int/iris/bitstream/handle/10665/274603/978924 1565639-eng.pdf

World Health Organizatión (2019). Global report on trends in prevalence of tobacco use 2000-2025. Recuperado de https://fundadeps.org/wpcontent/uploads/2020/02/Informe-OMS-consumo-tabaco-nivel-mundial.pdf

\section{AUTOR}

\section{Carlos A. Ballesteros Herencia}

Carlos A. Ballesteros Herencia es doctor en Ciencias de la Información (2012), licenciado en Periodismo (1995) y grado en Ciencias del Deporte (2011). Profesor en el grado de Periodismo de la Universidad de Valladolid. Gusta de analizar estadísticamente los marcos informativos (news frames), el engagement digital, la aplicación de las tecnologías a las campañas electorales y la responsabilidad de los medios de comunicación ante las drogas. Ha sido responsable de Comunicación de UPA-Castilla-La Mancha y director de La Voz de Albacete y El Espectador Crítico.

Orcid ID: https://orcid.org/0000-0002-8990-7949

Google Scholar: https://scholar.google.com/citations?user=ntT2zsIAAAAJ\&hl=en

Revista de Comunicación y Salud, 2020, Vol. 10, nº 1, pp. 43-66 
Bases para el tratamiento informativo de las drogas en los medios de comunicación

ResearchID: D-9772-2016

Research Gate: Carlos_Ballesteros9

Academia.edu: uva-es.academia.edu/Carlos_Ballesteros

Revista de Comunicación y Salud, 2020, Vol. 10, nº 1, pp. 43-66 